\title{
EVALUACIÓN DE ADITIVOS USADOS EN EL TRATAMIENTO DE ARCILLAS EXPANSIVAS
}

\section{EVALUATION OF ADDITIVES USED IN TREATMENT OF EXPANSIVE CLAYS}

Fecha de Recepción: 12 de Agosto de 2006

Fecha de Aprobación: 30 de Septiembre de 2006

\author{
Javier Fernando Camacho Tauta ${ }^{1}$ \\ Oscar Javier Reyes Ortiz ${ }^{2}$ \\ Catalina Mayorga Antolínez ${ }^{3}$ \\ Dolly Fernanda Méndez G. ${ }^{4}$
}

RESUMEN: Se utilizaron tres aditivos (cal, cenizas volantes y aceite sulfonado) para disminuir las propiedades de una arcilla altamente expansiva (bentonita), con el fin de determinar las ventajas técnicas de cada alternativa bajo las mismas condiciones y ensayo. Para tal fin la bentonita se mezcló con diferentes concentraciones de aditivo y se realizaron ensayos de caracterización física y mecánica que permitieron analizar el comportamiento de cada uno. Se encontró que las cenizas volantes requieren ser adicionadas en cantidades excesivas para lograr disminución del potencial de expansión a valores admisibles. Con la cal se encontró un valor óptimo del $10 \%$ ya que logró una reducción considerable en las propiedades expansivas de la bentonita. Los resultados obtenidos con el aceite sulfonado evidenciaron que la estabilización electroquímica que lleva a cabo, requiere de factores ambientales favorables para desarrollar su efecto.

PALABRAS CLAVE: Arcillas expansivas, estabilización, potencial de expansión, cal, ceniza volante, aceite sulfonado.

ABSTRACT: Three additives lime, fly ash and sulfonated oil were used to reduce the sweIling of a highly expansive clay (bentonite). This study was performed in order to determine technical advantages of each alternative under same test conditions with respect to limiting the expansive behavior of the clays. Bentonite was mixed using different concentrations in percentage per weight such as 10 to $50 \%$. Physical and mechanical tests were carried out to analyze the behavior of each mixture. It is necessary to use a considerable amount of fly ash to obtain an admissible swelling potential. The use of $10 \%$ of lime reduces swelling potential until acceptable values. Sulfonic oil requires environmental conditions to reach its electrochemical effects which have been seen in certain path tests exposed to own site temperature changes and ultraviolet radiation, improving the physical and mechanical properties.

KEYWORDS: Expansive clay, stabilization, swelling potential, lime, fly ash, sulfonated oil

1 Ing. Civil, MSc. Profesor Asistente Universidad Militar Nueva Granada. Bogotá, Colombia. Estudiante de Doctorado Universidad Técnica de Lisboa. jcamacho@umng.edu.co 2 Ing. Civil, MSc. Profesor Asistente Universidad Militar Nueva Granada. Bogotá, Colombia. Estudiante de Doctorado Universidad Politécnica de Cataluña. oreyes@umng.edu.co

3 Ing. Civil Universidad Militar Nueva Granada. Bogotá, Colombia

4 Ing. Civil Universidad Militar Nueva Granada. Bogotá, Colombia 


\section{INTRODUCCIÓN}

Los efectos de las arcillas expansivas han sido un problema que cada año se ve reflejado en cuantiosas pérdidas de dinero. Este problema se ha venido mitigando con la utilización de diversos métodos que principalmente se orientan hacia su estabilización, bien sea física o electroquímica, entre los cuales se destacan la cal, las cenizas volantes y los aceites sulfonados, que han sido estudio de investigaciones aisladas, pero no se encontraron investigaciones que evaluaran simultáneamente estas tres alternativas y bajo condiciones severas de expansión.

La utilización de la bentonita como arcilla de análisis, limita la investigación a condiciones de expansión elevadas que permiten mediante un trabajo en el laboratorio con ensayos de caracterización física y mecánica determinar cuál aditivo y dosificación se comporta mejor en el ambiente más crítico, para lo cual se utilizan diferentes concentraciones para cada aditivo.

Lara et al [12] utilizaron cal, yeso y poliuretano para estabilización de arcillas con un alto potencial expansivo. La investigación arrojó discusiones acerca de la importancia del conocimiento de la mineralogía del suelo para controlar su comportamiento expansivo, el cual fue analizado mediante métodos directos de laboratorio como la difracción por rayos $\mathrm{X}$, e indirectos como los límites de Atterberg, el potencial de cambio volumétrico y la actividad del suelo.

Akawwi [3] evaluó la estabilización con cal de suelos expansivos de Amman y empleó el método de la difracción por rayos $\mathrm{X}$ para identificar los minerales presentes en el suelo observando que el potencial de cambio volumétrico depende de la composición mineralógica y aumenta con el contenido de montmorillonita.

Bonilla [6] orientó la investigación hacia la utilización de las cenizas volantes como aditivo estabilizador por ser de fácil adquisición. Estudios realizados por Garzón et al [10], muestran el efecto que a nivel microscópico tiene el aceite sulfonado como consecuencia del desalojo de agua.

Resultados reportados por compañías de ingeniería (citados por Acresquim, 1993) muestran reducciones en el límite líquido y aumento en el CBR de subrasantes tratadas con Geo-Stab. Bateman reporta un limo arcilloso con límite líquido del $103 \%$ y CBR menor al $1 \%$ que al ser tratado presenta un límite líquido del $62 \%$ y CBR igual a $26 \%$. El tiempo de curado, incide también en la eficiencia del producto. Información reportada por Pilotajes Técnicos muestra como la presión de expansión tomada a diferentes días de tratamiento, va reduciéndose paulatinamente.

\section{MARCO CONCEPTUAL}

\section{A. Características físico-químicas de los suelos expansivos}

Los suelos expansivos son arcillas que sufren cambios de volumen en presencia del agua de acuerdo a las condiciones de origen, composición química y propiedades físicas. Las arcillas están básicamente compuestas por silicatos de aluminio que en algunos casos pueden ser reemplazados por silicato de magnesio o de calcio, cuyas partículas tienen una estructura químicamente definida, donde los átomos se precisan en láminas.

Existen tres grandes grupos de minerales que hacen parte de la formación básica de las arcillas: caolinitas, ílitas y montmorillonitas. Los suelos arcillosos no solamente son expansivos debido a la estructura química que hace parte de estos, sino que además la presencia de minerales como la montmorillonita permite mayores índices de hinchamiento. Dentro de la familia de las arcillas montmorilloníticas se encuentra la bentonita. Las características expansivas de diferentes tipos de bentonitas estudiadas alrede- 
dor del mundo se encuentran dentro de los siguientes valores:

- Límites líquidos mayores a $140 \%$, los cuales pueden llegar a ser de $650 \%$

- Límites plásticos entre $63.87 \%$ y $26.67 \%$

- Índices de plasticidad superiores a $80.6 \%$

- Actividad 7.79 y 4.36

- Contenidos de montmorillonita entre el 48 y $80 \%$

- Capacidad de intercambio catiónico con valores cercanos a $1.035 \mathrm{meq} / \mathrm{g}$

La bentonita que se utilizó en este trabajo posee las especificaciones técnicas, resumidas en las Tablas $1 \mathrm{y}$ 2. (Bentonitas Colombianas Ltda., 2002)

Tabla 1. Composición química de la bentonita, utilizada en las pruebas experimentales.

\begin{tabular}{|l|r|}
\hline Mineral & \% en masa \\
\hline $\mathrm{SiO}_{2}$ & 50.52 \\
\hline $\mathrm{Fe}_{2} \mathrm{O}_{3}$ & 7.27 \\
\hline $\mathrm{Al}_{2} \mathrm{O}_{3}$ & 17.25 \\
\hline $\mathrm{MgO}$ & 1.21 \\
\hline $\mathrm{NaO}_{2}$ & 2.48 \\
\hline $\mathrm{K}_{2} \mathrm{O}$ & 0.70 \\
\hline
\end{tabular}

Tabla 2. Análisis físico de la bentonita

\begin{tabular}{|ll|}
\hline Propiedades & Especificaciones \\
\hline Humedad a $105 \mathrm{C}(\%)$ & Máximo 12 \\
Viscosidad $600 \mathrm{rpm} / 300 \mathrm{rpm}$ & Mínimo 20/16 \\
Hinchamiento & Mínimo 23 \\
Granulometría, \% pasa malla 200 & Mínimo 80\% pasa malla 200 \\
$\mathrm{pH}$ & Máximo 11 \\
\hline
\end{tabular}

\section{B. Potencial de expansión de los suelos}

Muchos estudios coinciden en que los suelos con índices de plasticidad mayores a 35\% y un límite líquido superior a $60 \%$ son altamente expansivos, un ejemplo claro se aprecia en las Normas Colombianas Sismorresistentes de 1998 las cuales determinan como identificar los suelos expansivos. Ver Tabla 3.
Tabla 3. Evaluación del potencial de expansión de acuerdo con NSR-98

\begin{tabular}{|c|c|c|c|c|c|c|}
\hline $\begin{array}{c}\text { Potencial } \\
\text { de } \\
\text { expansión }\end{array}$ & $\begin{array}{c}\text { Expansión } \\
\text { Consolidómetro } \\
(\%)\end{array}$ & $\begin{array}{c}\mathrm{LL} \\
(\%)\end{array}$ & $\begin{array}{c}\mathrm{LC} \\
(\%)\end{array}$ & $\begin{array}{c}\mathrm{IP} \\
(\%)\end{array}$ & $\begin{array}{c}\text { Partículas } \\
\text { menores } \\
1 \mu \\
(\%)\end{array}$ & $\begin{array}{c}\text { Expansión } \\
\text { libre en } \\
\text { probeta } \\
(\%)\end{array}$ \\
\hline Muy alto & $>30$ & $>63$ & $<10$ & $>32$ & $>37$ & $>100$ \\
\hline Alto & $20-30$ & $50-63$ & $6-12$ & $23-45$ & $18-37$ & $>100$ \\
\hline Medio & $10-20$ & $39-50$ & $8-18$ & $12-34$ & $12-37$ & $50-100$ \\
\hline Bajo & $<10$ & $<39$ & $>13$ & $<20$ & $<17$ & $<50$ \\
\hline
\end{tabular}

\section{Estabilización de suelos expansivos}

La estabilización de un suelo es el proceso por el cual se mejoran las propiedades del mismo. Entre los aditivos usados en la estabilización de los suelos se encuentran los siguientes (Sowers, 1994):

- Aditivos que retienen humedad: sales comunes y cloruro de calcio.

- Aditivos resistentes a la humedad: agentes impermeabilizantes resinosos e hidrófugos, materiales bituminosos y asfaltos rebajados.

- Mezcla de suelo-cemento, en la mayoría de los casos se hace con cementos Pórtland.

- Cal y cemento: reacciona con el suelo químicamente permitiendo reacciones puzolánicas.

- Agentes dispersante: entre los cuales se encuentran el silicato de sodio y poli fosfato de sodio que reducen el límite líquido, el índice plástico y la permeabilidad.

A continuación se presenta una descripción de las sustancias utilizadas como aditivos en esta investigación, así como el efecto que producen sobre los materiales expansivos.

\section{Estabilización con cal}

La cal permite la reducción de límite líquido y el índice plástico, de tal manera que los cambios volumétricos también se disminuyan, así mismo produce reacciones químicas como intercambio catiónico, reacciones puzolánicas, incrementa la manejabilidad y permeabilidad, mejora las propiedades de resistencia, incrementa el límite de contracción y disminuye el índice plástico al igual que el límite líquido. (Braja, 2001) 


\section{Estabilización con cenizas volantes}

Las cenizas volantes son procedentes de la combustión en las centrales termoeléctricas. La composición de cada una de las clases de ceniza y el porcentaje de carbón encontrado en estas depende del proceso que se realice en cada una de las plantas de la termoeléctrica. Las cenizas son granos finos compuestos básicamente por silicatos, aluminios, cal libre y algunos óxidos que permiten una reacción puzolánica con el suelo que al igual que otras sustancias reduce el índice de expansión.

Una concentración del $25 \%$ de cenizas en el suelo puede resultar muy beneficiosa, afectando la granulometría, floculando los porcentajes de arcillas del suelo y reduciendo el límite líquido y el índice plástico del suelo. Las reacciones puzolánicas permiten incrementar la capacidad de soporte de la subrasante y con ello mejor las estructuras viales.

\section{Estabilización con aceite sulfonado}

Los aceites sulfonados y los lignosulfonatos se basan en la estabilización electroquímica, los cuales permiten un intercambio de iones entre el agua ionizada por el aceite y el suelo. Esta clase de aceites son de origen orgánico derivados de la combinación sulfuros y ácidos, aunque también hay otros que pueden ser parte de un derivado del grupo naftaleno del petróleo, como es el caso de Geo-stab. Los aceites lignosulfonatos reciben este nombre porque provienen de un compuesto orgánico como la lignina y se obtienen mediante el proceso del sulfito de madera.

Dentro de los aceites sulfonados que son usados para la estabilización del suelo se encontró que la empresa colombiana Acres Quím. Ltda, trabaja con un compuesto derivado de la fracción naftalénica de la hulla que se conoce con el nombre comercial de Geo-stab. Este es un líquido que se diluye en el agua permitiendo que actué en el suelo por medio de un intercambio catiónico, es de color pardo-rojizo, de densidad específica de 1.15 y con un pH de 1.0.
Geo-stab funciona por medio de agentes tensoactivos que rompen los enlaces electroquímicos y el agua fluye libremente, incrementando la resistencia al suelo y disminuyendo sus características expansivas. (Acresquim 1993).

El uso de aceites sulfonados y lignosulfonatos tiene como ventajas la reducción del agua contenida dentro de las partículas de suelo facilitando así la compactación, por medio de su acción catalizadora separa los iones $\mathrm{H}$ y $\mathrm{OH}$ liberando hidrógenos con cargas negativas y positivas y atrae iones de cargas positivas, además sus propiedades electrodinámicas reducen y fraccionan la doble capa aglomerando las partículas de suelo y disminuyendo la capacidad de hinchamiento.

Las dosificaciones del aceite dependen de su origen y concentración, pero es importante tener en cuenta que independientemente del producto que se utilice se sugiere un período de curado necesario para que el intercambio de iones se lleve a cabo. GeoStab por medio de una reacción tenso-activa donde se rompen los enlaces de arcillas-agua por medio de la ionización permite que los espacios intersticiales del suelo sean reducidos, haciendo un estrato menos permeable pero de mayor capacidad de soporte.

En el caso de Geo-Stab se recomienda una dosis de aplicación de $0.5 \%$, lo cual corresponde a 1 litro de Geo-Stab por 200 litros de agua, pero es importante conocer la fracción de finos que hace parte del suelo y la clasificación del suelo, así como el contenido de humedad natural y la humedad óptima del mismo. (Estavias, 2004).

\section{METODOLOGÍA EXPERIMENTAL}

Se realizaron ensayos de caracterización física y mecánica de cada una de las mezclas suelo-aditivo. Para el caso de las cenizas volantes y la cal, se mezcló de acuerdo con el peso de los sólidos. El 
aceite sulfonado Geo-stab se disolvió en agua para realizar la mezcla.

\section{A. Preparación y compactación de las muestras}

Se prepararon muestras a una humedad óptima y peso unitario seco máximo de acuerdo con el ensayo de compactación normal (ASTM D 698). Todas las muestras se sometieron a un proceso de curado en cuarto húmedo durante 15 días para las muestras con cal (CL) o ceniza volante (CV), mientras que para las muestras con aceite sulfonado (GS), se utilizó el tiempo recomendado por el fabricante (9o días) para buscar la completa reacción del aditivo.

Con el fin de compactar y ensayar muestras que tuvieran un contenido de agua apropiado se realizó el ensayo de compactación donde se obtuvo el peso unitario seco máximo de $1.55 \mathrm{t} / \mathrm{m} 3$, para una humedad óptima del $20 \%$

\section{B. Ensayos de caracterización física de las mezclas}

Después del periodo de curado, se realizaron ensayos para estudiar la variación de algunas propiedades físicas del material que son afectadas por la sustancia adicionada y que sirven de indicador de la naturaleza expansiva del material resultante. En este artículo se presentan los resultados correpondientes a los ensayos de límite líquido (LL) y e índice de plasticidad (IP). La ventaja de estos ensayos es que son sencillos y económicos de realizar.

\section{Ensayos de expansividad}

Se realizaron dos tipos de ensayo para determinar las características expansivas del material: Ensayo de expansión Lambe (PE) (ASTM D 4546) y el ensayo de índice de expansividad (IE) ) (ASTM D 4829).

El ensayo de Expansión de Lambe (PE) tiene como objetivo determinar la presión de expansión que una muestra de suelo es capaz de ejercer cuando es sumergida en agua, la prueba tiene una duración de dos horas y se requiere de un anillo de carga y de una cámara muy semejante a las que se usan en el ensayo de consolidación.

El ensayo del índice de expansión (IE) fue realizado de acuerdo con el procedimiento que se especifica en la Norma ASTM D 4829-95. Se sumerge la muestra de suelo durante 24 horas sometido a la acción de una presión vertical constante de 1 psi. Se expresa el índice de expansión como la relación que existe entre el incremento de la altura y la altura inicial de la muestra.

\section{ANÁLISIS DE RESULTADOS}

\section{Límite Líquido}

De acuerdo a los resultados obtenidos que se ilustran en la Figura 1, se observa que los tres aditivos reducen el límite líquido de la bentonita.

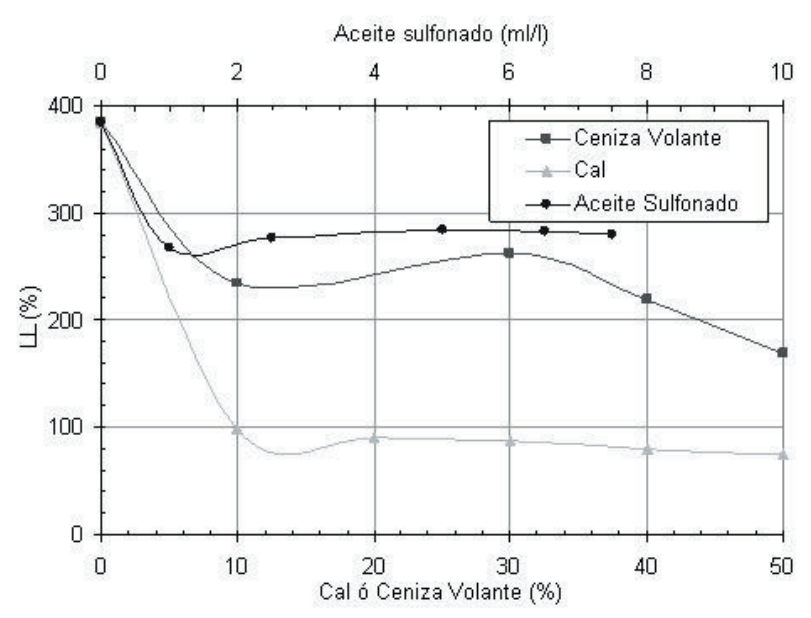

Figura 1. Variación en el límite líquido para cada dosificación

En el caso de las muestras tratadas con aceite sulfonado (GS), el límite líquido disminuye si se compara con el valor de la bentonita pura, teniendo en cuenta que este es del $384 \%$ y los límites de las mezclas de aceite sulfonado se encuentran en el rango de $266 \%$ a $285 \%$, aparentemente para ma- 
yores concentraciones no se logra mejores resultados.

En las mezclas (CV), se observa que a medida que la concentración de aditivo se incrementa el límite líquido reduce su valor, hasta llegar a un valor de $169 \%$ cuando la concentración de cenizas es del $50 \%$. La misma tendencia se ve reflejada en las mezclas $(\mathrm{CL})$, solo que en este caso las reducciones son muy significativas y a simple vista este aditivo reacciona mejor que los otros dos. Con una concentración del $10 \%$ de cal el límite líquido que se obtuvo fue del $98 \%$ y para concentraciones mayores los límites se van disminuyendo moderadamente.

De acuerdo con la clasificación de los suelos expansivos, el uso de los aditivos reduce el valor del límite líquido para la bentonita, pero para ninguno de los casos se llegó a un rango donde la expansión final fuera baja o marginal.

\section{2. Índice de Plasticidad}

Los resultados obtenidos se muestran en la Figura 2 , donde se ilustran los valores de índice de plasticidad para cada una de las mezclas. El índice de plasticidad tiene un comportamiento muy similar al caso anterior, aunque la mezcla (CL), al 50\% Ilega a pertenecer a los rangos de expansión baja. El aditivo que presentó el mejor comportamiento en este ensayo fue la cal, debido a que todas las concentraciones se encontraron entre los rangos de expansión marginal a baja.

Para las cenizas volantes es evidente que a mayor concentración de aditivo el valor del índice disminuye. El aceite sulfonado reacciona pero no de igual manera que en los casos anteriores, la tendencia es a mantenerse constante.

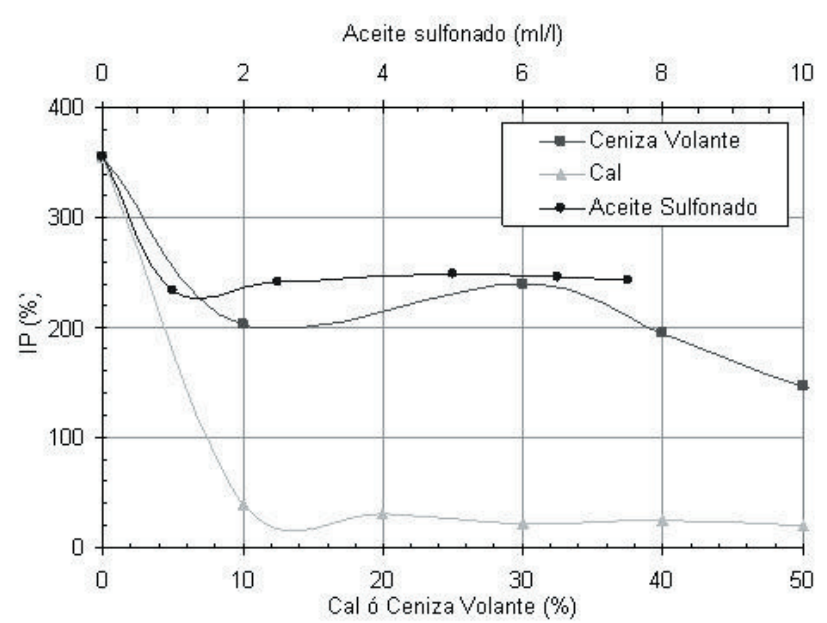

Figura 2. Índice de Plasticidad

\section{Presión de Expansión}

Luego de la compactación de las muestras y del tiempo de curado respectivo, se ensayó una muestra por cada una de las concentraciones de los tres aditivos. Se determinó que la presión de expansión para la bentonita es de $0.69 \mathrm{~kg} / \mathrm{cm}^{2}$, y según la Figura 3 se observa lo siguiente:

En el caso de las mezclas (CV), los valores de presión de expansión disminuyeron con respecto al valor de expansión para la bentonita pura.

Se destaca el temprano efecto de la cal que anula la presión de expansión.

En el caso de las mezclas (GS), las presiones fueron mayores a la presión de la bentonita pura y no existe una tendencia clara que permita determinar como se comporta el aditivo con relación a las concentraciones utilizadas. 


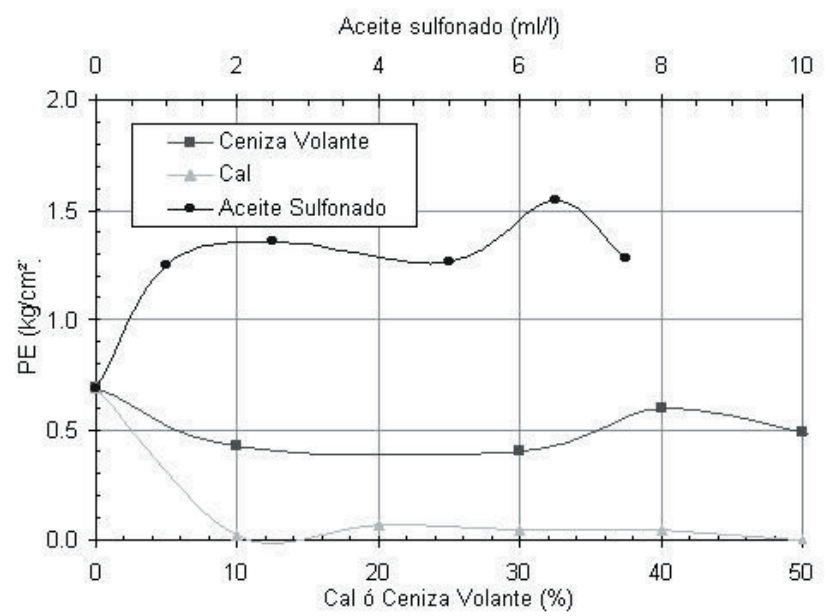

Figura 3. Presión de expansión para las diferentes mezclas

\section{4. Índice de Expansión}

La Figura 4 muestra que la cal es el aditivo más eficiente, ya que los índices de expansión para estas concentraciones clasifican en el rango de potencial de expansión muy baja.

\section{Incidencia de las condiciones de curado de las muestras (GS)}

Una explicación a los pobres resultados del GeoStab, fue encontrada en el trabajo desarrollado por Arellana, (2004). Este trabajo consistió en evaluar el Geo-Stab sobre muestras compactadas y curadas en laboratorio y en forma simultánea conformar un tramo de prueba en donde se aplicó el producto de acuerdo con las recomendaciones del fabricante. Posteriormente se extrajeron muestras del tramo de prueba y se realizaron los ensayos de laboratorio correspondientes. En la Figura 5 se muestran los resultados obtenidos en que se observa como las muestras curadas en condiciones de laboratorio no mejoraron sus valores de CBR mientras que en la Figura 6 se observa como las muestras curadas en condiciones ambientales obtenidas del tramo de prueba, aumentaron notablemente su resistencia. Similares resultados se encuentran para otras propiedades físicas y mecánicas.

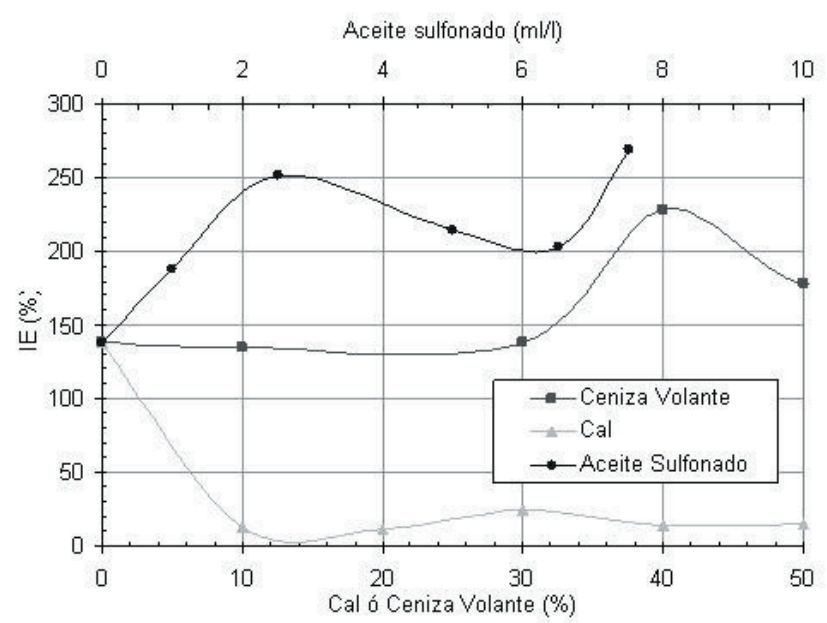

Figura 4. Índice de expansión para las diferentes mezclas

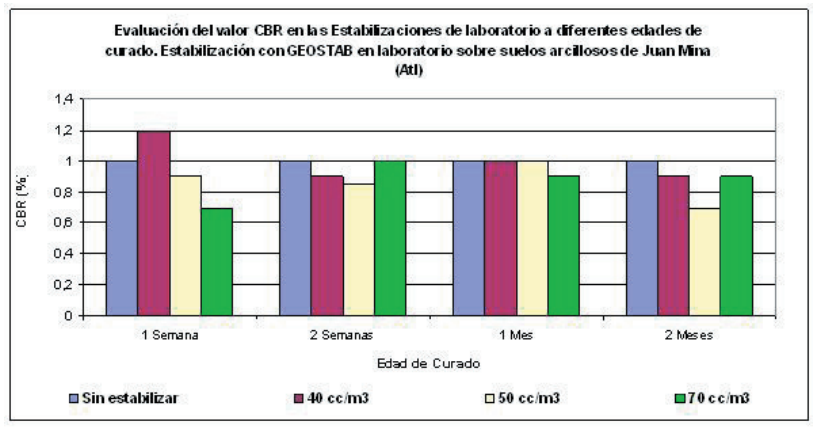

Figura 5. Valores de CBR para muestras curadas en laboratorio (Arellana, 2004)

Contrario a los resultados obtenidos en el laboratorio, en campo la reacción de Geo-stab, presenta reducciones del límite líquido y del índice de plasticidad, a partir de los 30 días de curado la tendencia a disminuirlos es proporcional al aumento en la dosificación y el tiempo de curado. (Arellana, 2002).

ProChemical Soil Stabilization [15] afirma que los suelos tratados en el laboratorio con estabilizadores químicos generalmente no presentan resultados concluyentes en la búsqueda de cambios profundos. Algunos de esos problemas pueden ser debido a que en laboratorio no se suelen simular las mismas condi- 
ciones ambientales del campo, las cuales aceleran el proceso electroquímico del aditivo. El efecto oxidante de los rayos ultravioleta favorece los efectos del producto, además del efecto catalítico del oxígeno presente entre las partículas del suelo.

Además del efecto mecánico que puede producir el tráfico sobre un tramo de prueba, se han observado cambios en los límites de consistencia, que solo pueden deberse al efecto de los estabilizadores químicos. Así mismo la Figura 6 muestra que en el mismo tramo de prueba sometido por consiguiente al mismo nivel de tráfico, la resistencia aumentó en mayor medida cuando se aumentó la dosificación.

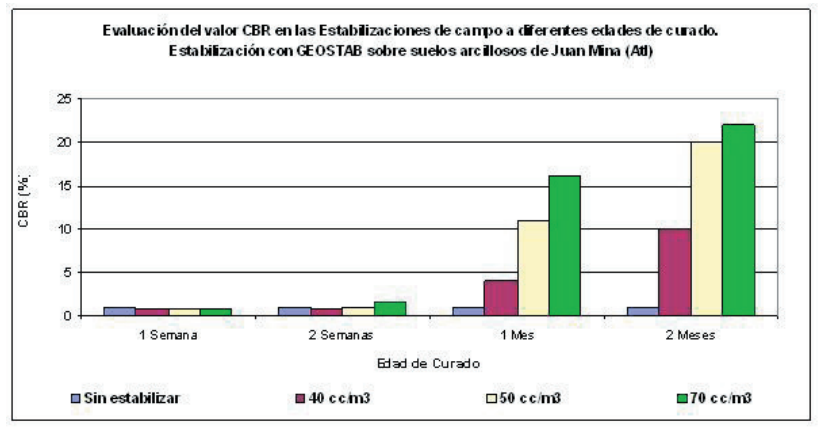

Figura 6. Valores de CBR para muestras curadas en campo (AreIlana, 2004)

\section{CONCLUSIONES}

Los resultados indican que la cal es el aditivo que reduce en mayor proporción las propiedades expansivas de la bentonita, desde las concentraciones mas bajas. Además en el caso del análisis de las propiedades de resistencia este aditivo es el que mejor desempeño tuvo, por lo tanto es el aditivo técnicamente más eficiente.

El porcentaje óptimo de cal en el tratamiento de un suelo expansivo es del $10 \%$, dicho porcentaje demostró que reduce significativamente las propiedades expansivas del material, no se incurre en demasiadas implicaciones técnicas si se necesita usar como alternativa de tratamiento.

Las cenizas volantes funcionan como aditivo inhibidor de las propiedades expansivas del material, pero a diferencia de la cal, este requiere ser adicionado en porcentajes excesivos, por lo menos para una arcilla tan expansiva como la bentonita, disminuyendo la viabilidad como alternativa de tratamiento.

En el caso del aceite sulfonado no existe una metodología adecuada de laboratorio que permita conocer la reacción de dicho aditivo. La ausencia de condiciones de curado similares a las condiciones de campo no permite una adecuada reacción electroquímica.

\section{AGRADECIMIENTOS}

Los autores agradecen el apoyo brindado por Acresquim Ltda y los Laboratorios de Ingeniería Civil de la Universidad Militar Nueva Granada, donde fueron realizados los ensayos reportados en este artículo.

\section{REFERENCIAS}

[1] ACRESQUIM LTDA. (1993) "Catálogo de productos", Bogotá.

[2] ADVANCED ENGINEERING GEOLOGY \& GEOTECHNICS. (2004) "Various aspects of expansive soils relevant to geoengineering practice". En: http://web. umr.edu

[3] AKAWWI, E., AL-KHARABSHEH, A. (2000) "Lime Stabilization Effects on geotechnical Properties of Expansive Soils in Amman Jordan". En: www.ejge.com. Volume 5

[4] ARELLANA, J. (2004). "Evaluación del efecto de la cal y el geostab en la estabilización de suelos arcillosos en Juan Mina". 172 p,: il. Tesis (Ingeniero Civil) . Universidad del Norte. Facultad de Ingenería Civil 
[5] BENTONITAS COLOMBIANAS LTDA. (2002) "Arcillas" En: www.bentocol.8m. com/index.htm

[6] BONILLA, B. (1996) "Estabilización de arcillas con cenizas volantes". Bogotá 99 p. : il., fot. Tesis (Ingeniero Civil). Universidad de los Andes. Facultad de Ingeniería

[7] BRAJA, M. (2001) "Principios de Ingeniería de Cimentaciones, México D.F, Thomson Editores, 862 p.

[8] DJEDID, A., BENKKOUCHE, A. Y MAMOUNE, A. (2001) "Identification and prediction of the swelling behaviour of some soils from the Tlemcen region of Algeria", 9 p.

[9] ESTAVIAS (2004) “Mantenimiento vial - estructura vial económicamente confiable, Estabilización de arcillas" Cap I. Colombia, 39p.

[10] GARZÓN F., DÍAZ, C. Y PÁEZ D. (2004) “Estabilización electroquímica con aceite sulfonado para arcillas representativas de la ciudad de Tunja". Paipa, Colombia: X Congreso Colombiano de Geotecnia

[11] KUMAR S, PURI, K BRAJA D. Y DEVKOTA B.C. (2001) "Geotechnical Properties Of Fly Ash and Lime: Fly Ash Stabilized Coal Mine Refuse". En: www.ejge. com. Volume 5

[12] LARA T., ZEPEDA J., Y CASTAÑO V. (1999) “A comparative study of the effectiveness of Different additives on the expansion behavior of clays". En: www. ejge.com. Volume 4.

[13] LÓPEZ, L. Y CASTAÑO V. (2001) "Time Dependency of CaO-Treated Expansive Soils". En: www.ejge. com

[14] MAYORGA, C. Y MENDEZ F., (2004) "Evaluación de métodos para la estabilización de arcillas expansivas" Proyecto de Grado (Ingeniero Civil). Universidad
Militar Nueva Granada. Facultad de Ingeniería

[15] PROCHEMICAL SOIL STABILIZATION INC. (1993) "Condor SS: An Electro-chemical Soil Stabilizer". Texas, $30 \mathrm{p}$.

[16] SOWERS, G. (1992) "Introducción a la Mecánica de Suelos y Cimentaciones", México D.F, Limusa S.A, $677 p$. 